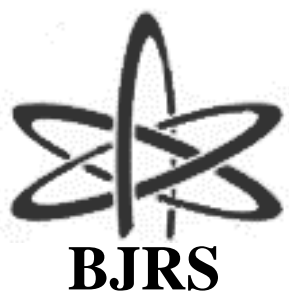
BRAZILIAN JOURNAL
$\mathrm{OF}$
RADIATION SCIENCES
03-1A (2015) 01-08

\title{
Calibração de detectores sólidos de traços nucleares do tipo CR-39 para medidas de Rn-222 no ar
}

\author{
C. D.Cazula ${ }^{\mathrm{a}}$; M. P. Campos ${ }^{\mathrm{a}}$; B. P. Mazzilli ${ }^{\mathrm{a}}$ \\ ${ }^{a}$ Gerencia de Metrologia das Radiações/Laboratório de Radiometria Ambiental, Instituto de Pesquisas Energéticas \\ e Nucleares, CEP, São Paulo-SP, Brasil \\ camilacazula@usp.br
}

\section{Resumo}

No método de detecção com detectores sólidos de traços nucleares, quando uma partícula pesada incide sobre a superfície do detector, provoca um desarranjo em sua estrutura molecular formando um traço. Uma das aplicações mais comuns desses detectores está na medição da concentração de Rn-222 no ar, um gás nobre radioativo, membro da série do U-238, emissor de partículas alfa e importante em estudos epidemiológicos para proteção dos indivíduos à radiação natural. Para determinar a concentração de Rn-222 no ar em um ambiente é necessário conhecer a densidade de traços (traços/cm2) na superfície do detector, o tempo de exposição e o fator de calibração. A determinação do fator calibração para os detectores CR-39 foi feita a partir da exposição destes a uma concentração conhecida de Rn-222. Para tanto, os detectores foram inseridos no interior de uma célula de Lucas adaptada e posteriormente expostos a uma concentração de Rn222 de $15 \mathrm{kBq} / \mathrm{m} 3$, por meio do aparelho RN-150 da Pylon Eletronics Incorporation, que possui uma fonte de Ra-226 e libera concentrações conhecidas de Rn-222. Foram realizadas seis determinações do fator de calibração, o valor médio obtido foi de 0,0534 \pm 0,0021 (traços/cm2 por $\mathrm{Bq} / \mathrm{m} 3$ dia). Os resultados são compatíveis com os valores da literatura para o mesmo tipo de detector e apresentaram boa reprodutibilidade.

Keywords: Radônio; Detectores de traços; Radiometria 


\section{INTRODUÇÃO}

A detecção passiva com o uso de detectores sólidos de traços nucleares é amplamente utilizada na determinação da concentração de Rn-222 no ar, um gás nobre radioativo, membro da série do U-238, emissor de partículas alfa e importante em estudos epidemiológicos para proteção dos indivíduos à radiação natural.

No método de detecção com detectores sólidos de traços nucleares, quando uma partícula pesada incide sobre a superfície do detector, provoca um desarranjo na sua estrutura molecular (traço). Entre os detectores de traços disponíveis comercialmente (fig. 1), o CR-39 é o que apresenta melhor qualidade ótica, já que é transparente e possibilita um contraste ideal entre o traço produzido e o próprio corpo do plástico. [1]

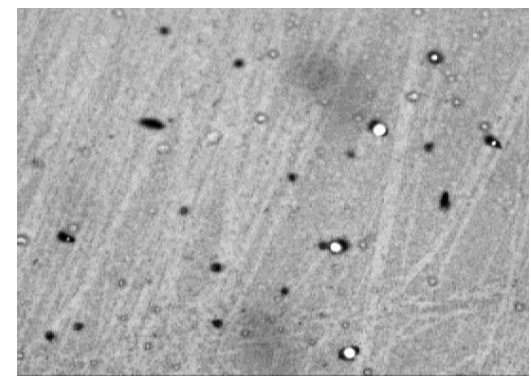

(a)

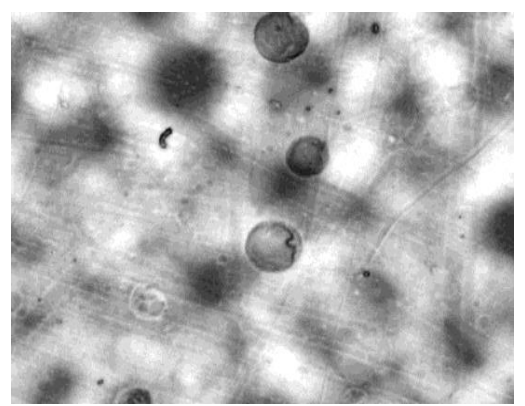

(b)
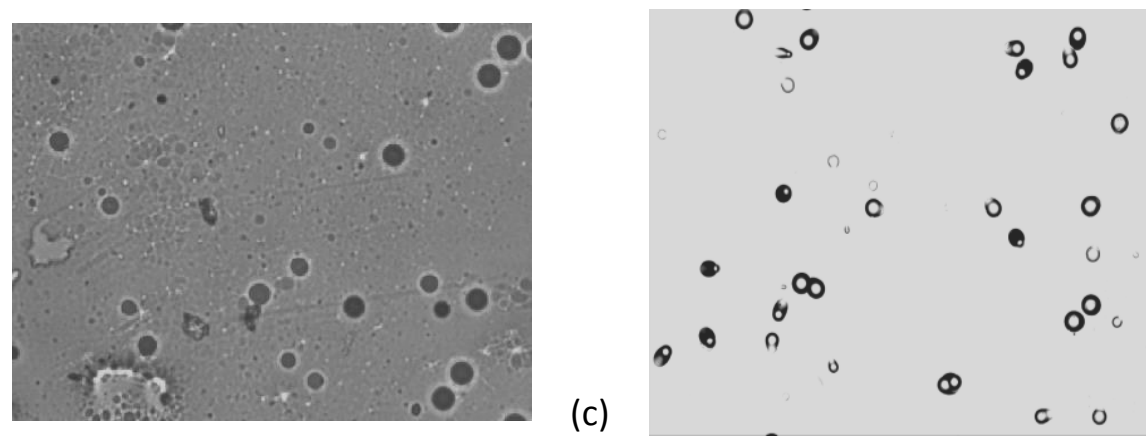

(d)

Figura 1. Traços na superfície de diferentes detectores de traços; (a) lr-115, (b) Makrofol, (c) Durolon, (d) Cr-39 
Para que os traços possam ser observados e analisados, é necessário aumentá-los, o que se consegue revelando-os com ataque químico ou eletroquímico. A análise do detector, por meio de microscopia ótica é realizada por métodos quantitativos, onde a determinação da quantidade de traços por unidade de área do detector fornece a densidade de traços (traços/cm2), parâmetro proporcional à concentração de Rn-222 [2].

Para calcular a concentração de Rn-222 é necessário conhecer a densidade de traços (traços/cm2), o tempo de exposição e o fator de calibração, relaciona a densidade de traços na superfície do detector e a concentração de radônio. Assim foram realizadas seis determinações do fator de calibração, a partir da seguinte equação [3].

$$
\mathrm{k}=\frac{\mathrm{D}}{\mathrm{C}_{\mathrm{Rn}} \cdot t}
$$

Onde:

$\mathrm{k}=$ fator de calibração (traços/cm 2 por $\mathrm{Bq} / \mathrm{m} 3 \mathrm{~d}-1)$;

$\mathrm{D}=$ Densidade de traços líquida (descontada a densidade relativa à radiação de fundo no detector) (traços/cm2);

C_Rn = Concentração de Rn-222 (Bq/m3);

$\mathrm{t}=$ tempo de exposição $(\mathrm{d})$;

\section{MATERIAIS E MÉTODOS}

Para a determinação do fator de calibração, um detector de traços nucleares do estado sólido (CR-39) idêntico aos utilizados nas medidas de Rn-222 no ar, foi inserido em uma célula de Lucas adaptada e exposto ao Rn-222 por meio do aparelho RN-150 da Pylon Eletronics Incorporation. Este aparelho contém uma fonte sólida calibrada de Ra-226 e opera com uma bomba de 
vácuo, liberando uma concentração conhecida de Rn-222 de 15,2 kBq m-3 para o recipiente que contém o detector a ser calibrado (fig. 2).

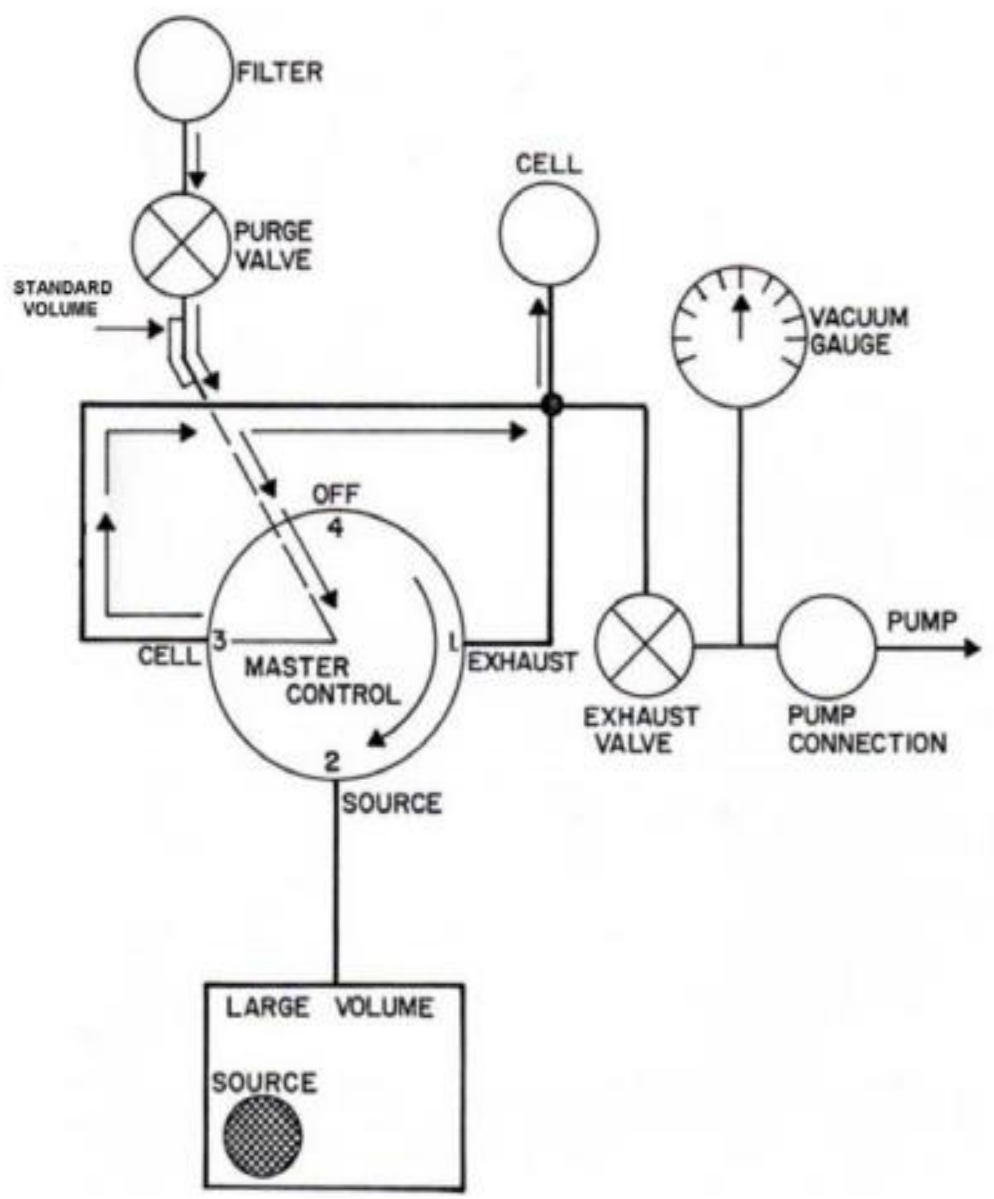

Figura 2. Esquema do sistema RN-150

A célula de Lucas, após o processo de emanação, fica selada por cerca de 4 dias, o que corresponde a aproximadamente uma meia-vida do Rn-222, dessa forma, o gás e os seus descendentes estarão presentes no ambiente confinado da célula de Lucas, sensibilizando o detector. $[4,5]$

Após a exposição os detectores sofrem ataque químico com solução de hidróxido de potássio, $30 \%$ em massa, durante 5,5 horas a $80^{\circ} \mathrm{C}$. Uma vez revelados, os detectores foram observados num microscópio óptico ZEISS modelo AxioImager para luz transmitida com aumento de 10x. O equipamento está ligado a uma câmara de vídeo marca Zeiss ICc-1 e acoplado a um mi- 
crocomputador com um monitor de 29" marca HP . O fator de calibração é então determinado por meio da equação 1 .
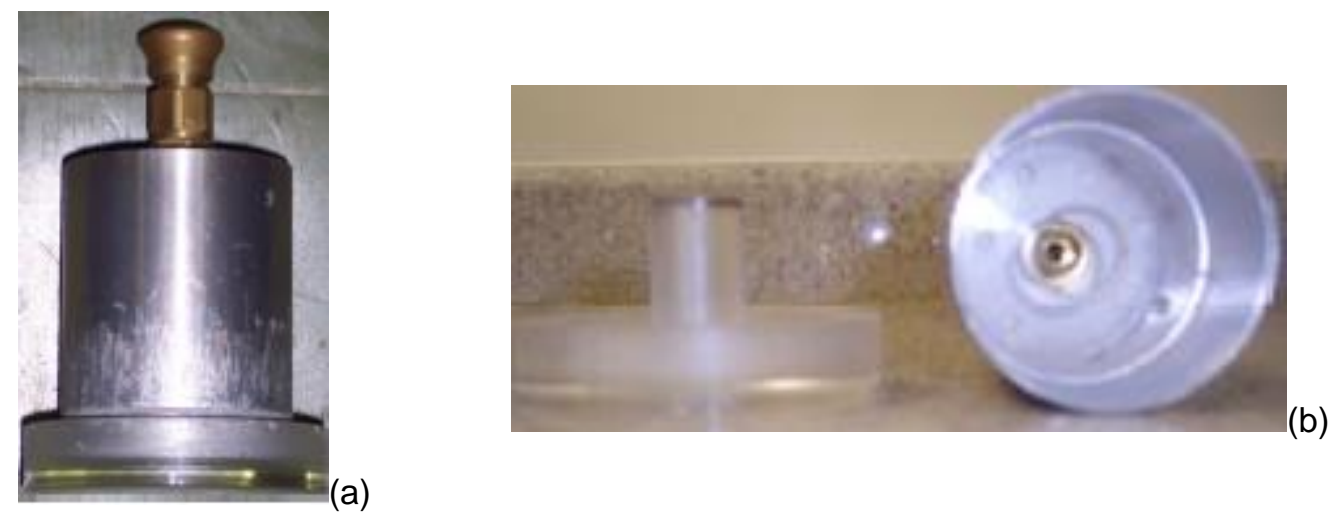

Figura 4.6: Célula de Lucas adaptada. (a) fechada (b) aberta

O CR-39 utilizado possui numeração e demarcação com 2,38 cm², chamada de área útil do detector, onde são realizadas as imagens para leitura dos traços, embora em toda superfície seja possível observar e quantificar os traços (fig. 3).

A radiação de fundo em detectores CR-39 varia de lote para lote, ou até mesmo de em diferentes regiões de uma mesma placa com vários detectores. Então, a radiação de fundo foi determinada individualmente para cada um dos detectores, onde antes da exposição, foi aplicado uma fita de polietileno (que permite a remoção sem deixar resíduos de adesivo), a fim de barrar as partículas alfa proveniente do Rn-222 e seus filhos. Essa área $\left(1,68 \mathrm{~cm}^{2}\right)$ então passou pelos mesmos procedimentos de leitura, determinando assim a radiação de fundo de cada detector.

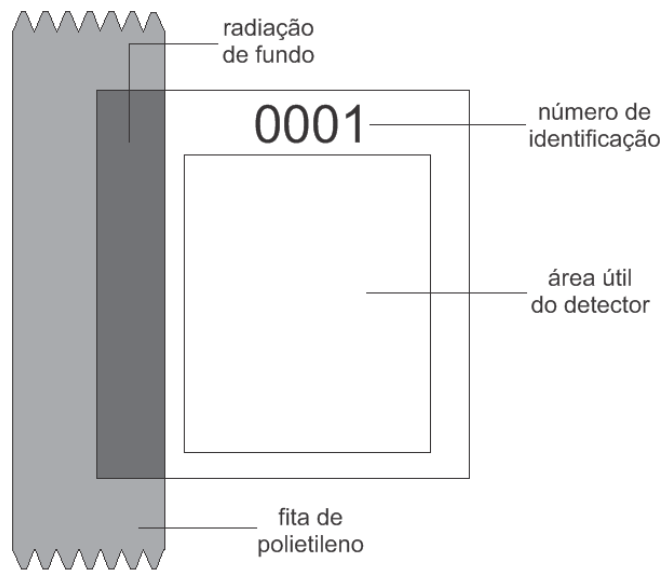

Figura 3. Esquema do detector de traços CR-39 
O valor médio da radiação de fundo obtida foi de $16,88 \pm 0,86$ traços/cm2, valores compatíveis com a literatura para o mesmo tipo detector e fabricante [6].

\section{RESULTADOS E DISCUSSÕES}

Foram realizadas seis determinações do fator de calibração (tab 1). O valor médio obtido foi de $0,0534 \pm 0,0021$ (traços/cm2 por $\mathrm{Bq} / \mathrm{m} 3 \mathrm{dia}$ ). Os resultados são compatíveis com os valores da literatura para o mesmo tipo de detector e apresentaram boa reprodutibilidade.

Tabela 1. Fator de calibração

\begin{tabular}{ccc}
\hline Calibração & Tempo de exposição (dias) & $\begin{array}{c}\text { Fator de Calibração } \\
\left(\text { traço/cm } \mathbf{c m}^{\mathbf{2}} \mathbf{p o r} \mathbf{B q} / \mathbf{m}^{\mathbf{3} \cdot \mathbf{d})}\right.\end{array}$ \\
\hline 1 & 3,987 & $0,0505 \pm 0,0001$ \\
2 & 3,984 & $0,0526 \pm 0,0001$ \\
3 & 3,854 & $0,0566 \pm 0,0001$ \\
5 & 4,041 & $0,0525 \pm 0,0002$ \\
6 & 3,313 & $0,0541 \pm 0,0003$ \\
& 3,106 & $0,0549 \pm 0,0003$ \\
\hline
\end{tabular}

\section{CONCLUSÕES}

Embora o fator de calibração para detectores de traços tipo CR-39 esteja disponível na literatura e seja fornecido pelos fabricantes, estabelecer o fator de calibração experimentalmente, proporciona uma verificação de conduta de todo procedimento, tal como revelação e leitura dos detectores. A prática de calibração utilizada, assim como o método para radiação de fundo, apre- 
senta resultados compatíveis com os valores da literatura para o mesmo tipo de detector e apresentaram boa reprodutibilidade $[3,8,9]$. O sistema demonstrou ser adequado e os resultados obtidos serão aplicados em futuras medições de Rn-222 no ar.

\section{AGRADECIMENTO}

Um dos autores (C. D. Cazula) agradece a Fundação de Amparo a Pesquisa de São Paulo pela concessão da bolsa de mestrado. (Processo FAPESP 2013/01841-9) 


\section{REFERÊNCIAS}

1. Paulo, S.R. "Dosimetria ambiental de Rn-222 e filhos: Medida da eficiência absoluta do CR39 levando-se em conta os efeitos do plate-out e fatores ambientais",. Tese (Doutorado) Universidade Estadual de Campinas. Campinas: 1991

2. Nikezic, D., "Determination of detection efficiency for radon and radon daughters with $C R$ 39 track detector - a Monte Carlo study"Nucl. Instruments Methods in Physics Research pp 406-414 (1994)

3. Mayya Y. S., Eappen K. P., Nambi K. S. V., "Methodology for mixed field inhalation dosimetry in monazite areas using a twin-cup dosimeter with three track detectors", Rad. Prot. Dosim., 77(3), pp.177-184 (1998).

4. PYLON ELECTRONICS INCORPORATION Pylon model RN-150 Manual Instruction, 1992.).

5. Campos, M. P. ; Matins, E.W. . "Calibration of the solid state nuclear track detector CR-39 for radon measurements.” In: International Nuclear Atlantic Conference, 2007, Santos. Proceedings International Nuclear Atlantic Conference, 2007. v. E05. p. 1-4.

6. Mishra,R, Orlando C., Tommasino L., Tonnarini S., Trevisi, "A better understanding of the background of CR-39 detectors”, Radiation Measurements, v.40 p. 325-328, 2005

7. Manocchi, F H ; Campos, M P ; Dellamano, J C ; Silva, G M ., "Radon exposure at a radioactive waste storage facility.”, Journal of Radiological Protection, v. 34, p. 339-346, 2014 